\section{Closure to "Uplift Capacity of Anchors in Layered Sand Using Finite-Element Limit Analysis: Formulation and Results" by Paramita Bhattacharya and Jyant Kumar}

DOI: 10.1061/(ASCE)GM.1943-5622.0000560

\section{Paramita Bhattacharya ${ }^{1}$ and Jyant Kumar ${ }^{2}$}

${ }^{1}$ Assistant Professor, Civil Engineering Dept., Indian Institute of Technology, Kharagpur 721302, India. E-mail: paramita@ civil.iitkgp.ernet.in

${ }^{2}$ Professor, Civil Engineering Dept., Indian Institute of Science, Bangalore 560012, India (corresponding author). E-mail: jkumar@civil.iisc.ernet .in

The authors thank the discusser for pointing out a few typographic errors, which occurred because some of the figures used for the final production were slightly different from those used for correcting the proofs. Different versions of the files, unfortunately, created the difference at the time of final production.

In Fig. 4, (1) the top-most solid and dashed lines are meant for $H / B=7$, (2) the bottom-most solid and dashed lines correspond to $H / B=3$, and (3) the intermediate lines are associated with $H / B=4$ 6 from bottom to top.

The legend of Fig. 1(d) remains exactly the same as that kept earlier for Fig. 1(c); (1) hollow circular markers are meant for a circular anchor plate, and (2) solid square markers are meant for a strip anchor.

In Figs. 6(a-c), the top set of curves is meant for $H / B=6$, and the bottom set of curves is associated with $H / B=4$. For Figs. 6 ( $a$ and $b$ ), there is no change in the definition of the axes; the vertical axis provides the values of $P_{u} /(A \gamma B)$. One can cross check it by comparing the results in 2(a) and 6(a and b). Note that, for $H / B=4$ and 6 , if the results of Fig. 2(a) are multiplied with $H / B$, then Figs. 6(a and b) will be obtained. For Figs. 6(a and b), $P_{u} /(A \gamma B)$ was determined with $q=$ 0 but $\gamma \neq 0$, and for Fig. 6(c), $F_{q}$ was obtained with $q \neq 0$ but $\gamma=0$. For the curves in Fig. 6(c), the hollow triangular marker is meant for the upper-bound results provided by Kumar (2003), and the remaining markers all correspond to the published lower-bound results by finite-element limit analysis.

Fig. 5 shows the results for $H / B=5$.

The equilibrium equations that were followed for an axisymmetric problem are

$$
\begin{gathered}
\frac{\partial \sigma_{r}}{\partial r}+\frac{\partial \tau_{r z}}{\partial z}+\frac{\sigma_{r}-\sigma_{\theta}}{r}=0 \\
\frac{\partial \tau_{r z}}{\partial r}+\frac{\partial \sigma_{z}}{\partial z}+\frac{\tau_{r z}}{r}=\gamma
\end{gathered}
$$

The analysis has been done for $\gamma_{1} / \gamma_{2}=1, \gamma_{1} / \gamma_{2}=1.4, \gamma_{2} / \gamma_{1}=$ 1 , and $\gamma_{2} / \gamma_{1}=1.4$; a typing error appears in the first paragraph of the "Variation of Uplift Factors" section of the original paper.

No additional lines need to be added at the location of the anchor, because the position of the anchor plate is shown quite clearly in Fig. 1(a). The anchor plate is placed on a loose sand layer, and the values of the soil unit weight and the frictional angle for the soil layer below the anchor plate do not affect the uplift capacity.

The pullout capacity factor $F_{q}$ was determined for $\gamma=0$ and $q \neq$ 0 , and it can be defined by the following:

$$
F_{q}=\frac{P_{u}}{A q}=\frac{p_{u}}{q}
$$

The variations of $F_{q}$ with $H_{\text {dense }} / H$ for $\phi_{1}=20^{\circ}, \phi_{2}=45^{\circ}$ and $\phi_{1}=45^{\circ}, \phi_{2}=20^{\circ}$ were obtained, and these values were compared with that reported by the discusser. The comparison between the results is presented in Fig. 1 of this paper. Note that the two solutions compare quite closely.

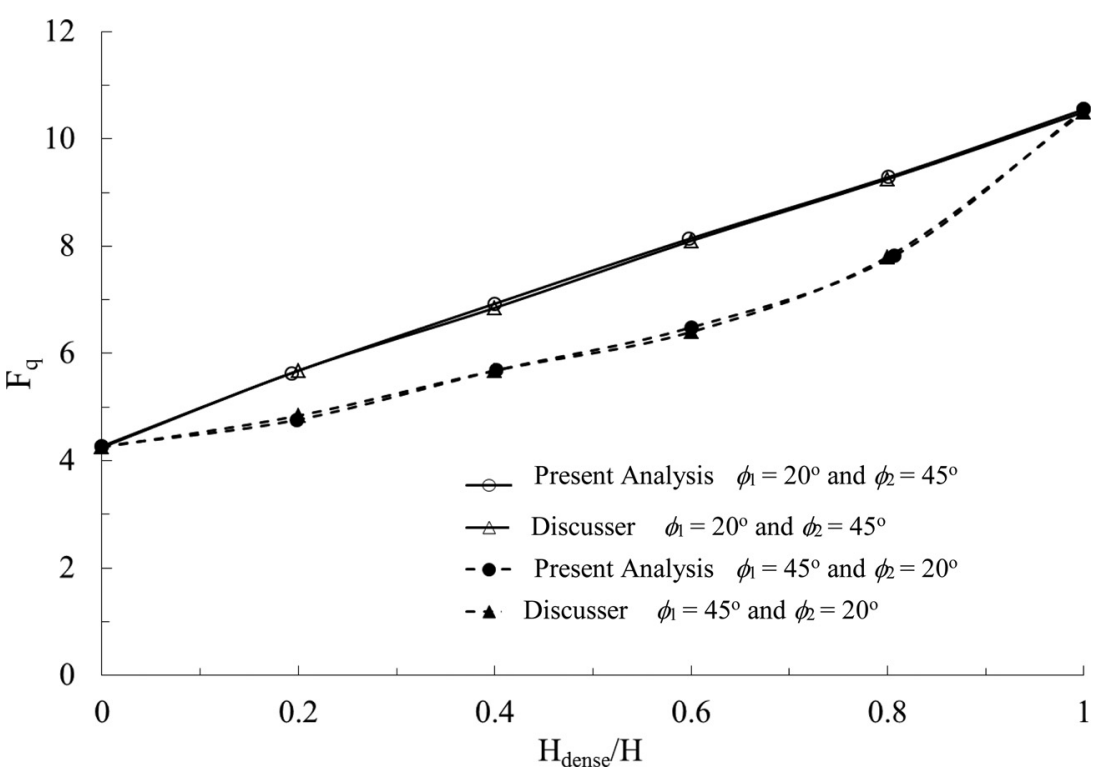

Fig. 1. Comparison between the present solution and that reported by the discusser for a strip anchor with $H / B=5$ 
By considering the principle of superposition, one can compute the pullout capacity of the anchor plate in soil mass by using the following equation:

$$
P_{u}=A \gamma H F_{\gamma}+A q F_{q}
$$

Note that for computing $F_{q}$, the value of soil unit weight needs to be 0 and it should not be for $\gamma_{1} / \gamma_{2}=1$, as pointed out by the discusser.

However, for sand material, it is quite unlikely that the value of $\phi$ will become less than $30^{\circ}$. Therefore, in the published paper, the lowest value of $\phi$ was considered to be $30^{\circ}$.

At the end of the "Domain, Stress Boundary Conditions, and Finite-Element Mesh" section of the original paper, the 100,000 means 1 lac only.

\section{Concluding Remarks}

This study determines numerically the ultimate uplift capacity of anchors in layered sand. At present, the analysis is based on the assumption that the principle of linear superposition remains valid. One can easily perform the analysis by taking into account the actual values of unit weights of the two layers along with surcharge pressure to obtain the true magnitude of the collapsed load without making this assumption.

\section{References}

Kumar, J. (2003). "Uplift resistance of strip and circular anchors in a two layered sand." Soils and Foundations, 43(1), 101-107. 